\title{
Pinning-Induced Formation of Vortex Clusters and Giant Vortices in Mesoscopic Superconducting Disks
}

\author{
I. V. Grigorieva, ${ }^{1, *}$ W. Escoffier, ${ }^{1}$ V. R. Misko, ${ }^{2}$ B. J. Baelus, ${ }^{2}$ F. M. Peeters ${ }^{2}$ L. Y. Vinnikov, ${ }^{3}$ and S. V. Dubonos ${ }^{3}$ \\ ${ }^{1}$ School of Physics and Astronomy, University of Manchester, Manchester M13 9PL, United Kingdom \\ ${ }^{2}$ Departement Fysica, Universiteit Antwerpen, Groenenborgerlaan 171, B-2020 Antwerpen, Belgium \\ ${ }^{3}$ Institute of Solid State Physics, Russian Academy of Sciences, Chernogolovka 142432, Russia
}

(Received 8 November 2006; published 3 October 2007)

\begin{abstract}
Merged, or giant, multiquanta vortices (GVs) are known to appear in very small superconductors near the superconducting transition due to strong confinement of magnetic flux. Here we present evidence for a new, pinning-related, mechanism for vortex merger. Using Bitter decoration to visualize vortices in small $\mathrm{Nb}$ disks with varying degrees of disorder, we show that confinement in combination with strong disorder causes individual vortices to merge into clusters or even GVs well below $T_{c}$ and $H_{c 2}$, in contrast to welldefined shells of individual vortices found in the absence of pinning.
\end{abstract}

DOI: 10.1103/PhysRevLett.99.147003

Mesoscopic superconductors, i.e., such that they can accommodate only a small number of vortices, are known to exhibit complex and unique vortex structures due to the competition between surface superconductivity and vortex-vortex interactions [1-3]. For mesoscopic disks, theoretical studies found two kinds of superconducting states: a giant vortex $(\mathrm{GV})$, i.e., a circular symmetric state with a fixed value of angular momentum that can carry several flux quanta, and multivortex states (MVS) with an effective total angular momentum corresponding to the number of vortices in the disk (vorticity $L$ ) (see, e.g., Ref. [1]). Experimentally one can distinguish between a single-core GV and a MVS using, e.g., the multiple-smalltunnel-junction method [2]. GVs have also been inferred from an experimental observation that vortices in perforated $\mathrm{Al}$ films merge into large flux spots close to the superconducting transition temperature $T_{c}$ [4]. In another recent experiment [3], Bitter decoration was used to directly visualize MVS in small $\mathrm{Nb}$ disks, and circular symmetry was found to lead to the formation of concentric shells of vortices, similar to electron shells in atoms. Analysis of different vortex configurations revealed the rules ("periodic law") of shell filling, as well as "magicnumber" configurations corresponding to commensurability between the shells.

Here we show that, while weak pinning in mesoscopic disks only leads to distortions in symmetric shell configurations, the presence of strong disorder changes the situation dramatically. Using Bitter decoration to visualize vortices in small disks etched in the surface of $\mathrm{Nb}$ single crystals and numerical simulations to analyze the role of disorder, we find that the interplay between confinement and pinning results in the formation of clusters or even GVs in relatively large samples and at temperatures well below $T_{c}$, due to selective enhancement of pinning strength by disks' boundaries. Depending on the disk's size and the applied field, we find that either all vortices merge into a single cluster or GV (for small $L$ ) or clusters or GVs
PACS numbers: 74.78.Na, 74.20.De, 74.25.Op, 74.25.Qt

coexist with singly-quantized vortices, for larger $L$. No clustering or GV formation was found for the same disorder in macroscopic samples.

Samples for this study were prepared from bulk $\mathrm{Nb}$ single crystals using $e$-beam lithography and reactive etching $\left(\mathrm{CF}_{4}\right.$ with added oxygen) followed by high-vacuum annealing (at $<10^{-8}$ torr) at temperatures $\sim 750^{\circ} \mathrm{C}$, to remove gaseous contaminants. This produced large arrays of disks - 0.5-1.4 $\mu \mathrm{m}$ high, 2-5 $\mu \mathrm{m}$ diameter-on the surface of $\sim 0.5 \mathrm{~mm}$ thick $\mathrm{Nb}$ crystals [see upper-right inset in Fig. 1(a)]. The array geometry and decoration details were the same as in our previous study [3], i.e., a whole array of over 300 disks was decorated in each experiment after field cooling to $\sim 1.8 \mathrm{~K}$, allowing us to obtain simultaneous snapshots of up to a hundred vortex configurations in nominally identical disks. To assess the degree of disorder before and after annealing, the samples were decorated straight after etching, and then after annealing for $t=1,2,4$, and $6 \mathrm{~h}$, and magnetization and resistivity were measured at $t>2 \mathrm{~h}$. This revealed pronounced changes in $T_{c}$ and bulk pinning, consistent with the presence of solute oxygen atoms in interstitial positions [5,6]: Interstitial oxygen is known to greatly reduce $T_{c}$ even in small concentrations [5] and is the only impurity that is greatly affected by the above annealing regimes due to its short diffusion times [6] (for the used sample dimensions, annealing for $6 \mathrm{~h}$ at $750^{\circ} \mathrm{C}$ is sufficient to remove interstitial oxygen completely). Therefore, by varying the annealing time we were able to achieve partial removal of solute oxygen thereby changing the degree of disorder (in the form of $\sim 10-50 \mathrm{~nm}$ nonsuperconducting inclusions, where oxygen atoms cluster around point defects, dislocations, and other imperfections [5]). Accordingly, no vortices were observed either in the bulk of the crystals or inside the disks for $t<2 \mathrm{~h}$, as contamination rendered the samples nonsuperconducting at relevant $T$, but annealing for $t \geq 2 \mathrm{~h}$ recovered superconductivity over the entire crystals, with the amount of disorder gradually decreasing 


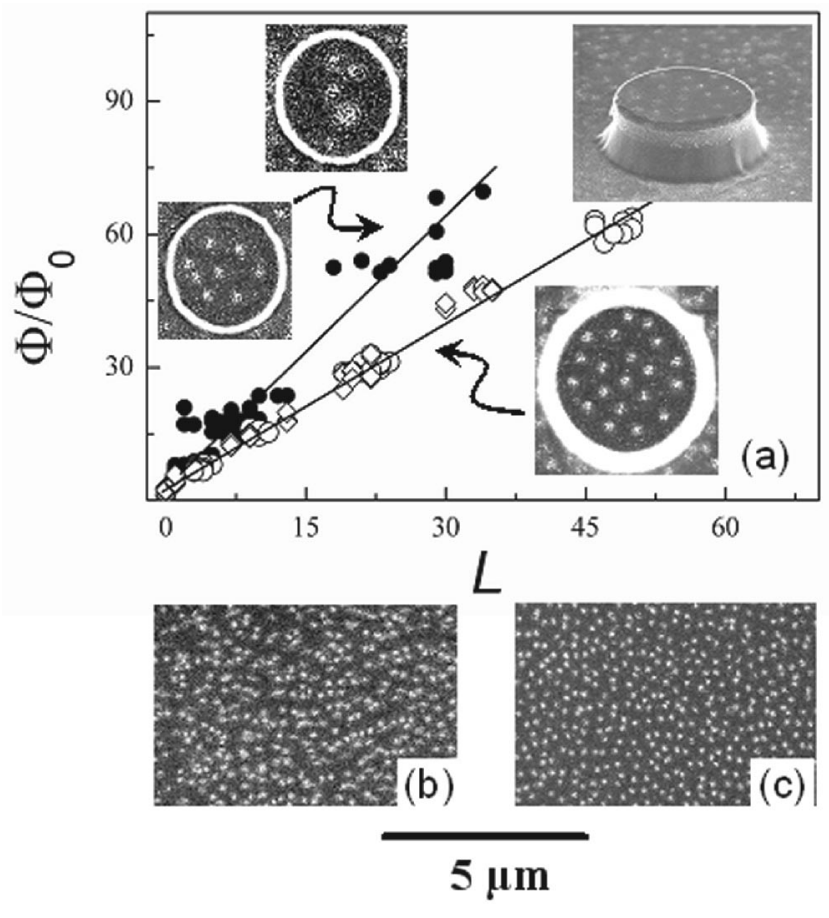

FIG. 1. (a) Main panel: Diamagnetic response of single-crystal-based disks with weak $(\bigcirc)$ and strong $(O)$ disorder; data for individual thin-film disks from Ref. [5] included for comparison $(\diamond)$. Solid lines are guides to the eye. Top-left and bottom-right insets show vortex configurations in disks with strong and weak disorder, respectively ( $H=70 \mathrm{Oe})$. Top-right inset: Vortices in and around a $1.4 \mu \mathrm{m}$ high disk decorated after field cooling in $H=60$ Oe. (b),(c) Vortex distributions in the bulk $(\sim 100 \mu \mathrm{m}$ away from mesoscopic disks) of $\mathrm{Nb}$ crystals with strong and weak disorder, respectively.

with increasing $t$; see Figs. 1(b) and 1(c). The disordered and very inhomogeneous vortex distribution in Fig. 1(b) is consistent with the presence of many pins with sizes $\geq \xi$ [7]. Longer $t$ resulted in a homogeneous, although still disordered, vortex structure [Fig. 1(c)], very similar to that in macroscopic $\mathrm{Nb}$ films from our previous study [3].

Figure 1(a) (bottom-right inset) shows a typical vortex configuration in disks with weak disorder. Clearly, the effect of vortex confinement in single-crystal-based disks is very similar to that in individual thin-film disks studied in [3]; i.e., confinement has a mitigating effect on disorder in that, despite the presence of weak pinning, vortices form nearly perfect shell configurations [e.g., state $(1,6,13)$ in Fig. 1(a)]. Furthermore, both systems show identical diamagnetic response [see $\Phi / \Phi_{0}$ vs $L$ curves in Fig. 1(a), where $\Phi=H S$ is the flux through the disk's area $S, H$ the applied magnetic field, and $\Phi_{0}$ the flux quantum] and similar vortex shell states. Details of the observed behavior will be published elsewhere. The important implication for the present study is that the effect of vortex confinement in single-crystal-based disks is identical to that in individual thin-film disks, even though in the former case only a small fraction of the total vortex length is subject to the additional interaction with the surface. Moreover, the response was found to be independent of the disk's height, in agreement with theoretical predictions [8]. In contrast, in the case of strong disorder [as in Fig. 1(b)], instead of mitigating pinning as above, confinement was found to selectively enhance the effect of pins, ultimately leading to merger of vortices into clusters or GVs, as demonstrated below. The most striking features of vortex configurations in this case are, first, that some of the "vortices" inside the disks appear to be significantly larger than vortices in the bulk or in weak-disorder disks and, second, that there are on average noticeably fewer vortices in these disks, compared to the number expected for a given value of $\Phi / \Phi_{0}$ or found in weak-disorder samples, i.e., they show a stronger diamagnetic response and a significant variation in the observed value of $L$ for the same $\Phi / \Phi_{0}$, as demonstrated in the main panel of Fig. 1(a). Indeed, a wide range of $L$ values is observed for any given $\Phi / \Phi_{0}$ (e.g., $L=2-6,8$, and 9 for $\Phi / \Phi_{0}=17-19$ while only $L=12$ and 13 are found for this flux interval in weak-disorder disks) and even the maximum observed $L$ is $25 \%-30 \%$ lower than for the weak-pinning disks, indicating that strong disorder somehow facilitates expulsion of extra vortices. The two left insets of Fig. 1(a) show vortex images observed in the same experiment $(H=70 \mathrm{Oe})$ in identical $3 \mu \mathrm{m}$ disks. Vortices in the bottom image form a slightly distorted shell configuration $(1,7)$ while a disordered pattern of only 5 vortices is seen in the second disk of exactly the same area, with one of the vortices having a $\sim 2.5$ times larger diameter compared to the rest. Indeed, identifiable shell configurations containing identical (small) vortices were seen only rarely in these samples while most disks contained a combination of small and large vortices in a disordered pattern or just a single large vortex. Figure 2 shows typical observations for 2,3 , and $5 \mu \mathrm{m}$ disks at $\Phi / \Phi_{0}$ corresponding to different $L_{\max }$ (i.e., maximum number of vortices found in disks with only small, singly-quantized, vortices). For $L_{\max }=3$ most disks contained 2 or 3 standard-size vortices (top image); i.e., the diameter of a cluster of $\mathrm{Fe}$ particles "decorating" a vortex was the same as for vortices in the bulk and in weak-disorder disks, but a significant proportion of disks showed only one vortex of about $80 \%$ larger diameter (bottom image). For $L_{\max }=9$ only $10 \%$ of the disks showed configurations of small vortices (top image) while most of the rest contained a combination of several small and 1 or 2 large vortices (as in bottom image) and a few disks showed only one very large vortex, as in the bottom image for $L_{\max }=11$. As the vorticity increased above $L_{\max }=11$, very few disks contained only small vortices while a typical configuration was a combination of small vortices and a number of large vortices of several different diameters, as in the image for $L_{\max }=36$.

To understand the nature of large vortices observed in disks with strong disorder, we recall that there is an excellent correlation between the size of vortex images in decoration experiments and the "magnetic" size of individual vortices, $\propto \lambda$ [7]. This allows us to identify the observed large vortices as several singly-quantized vortices 


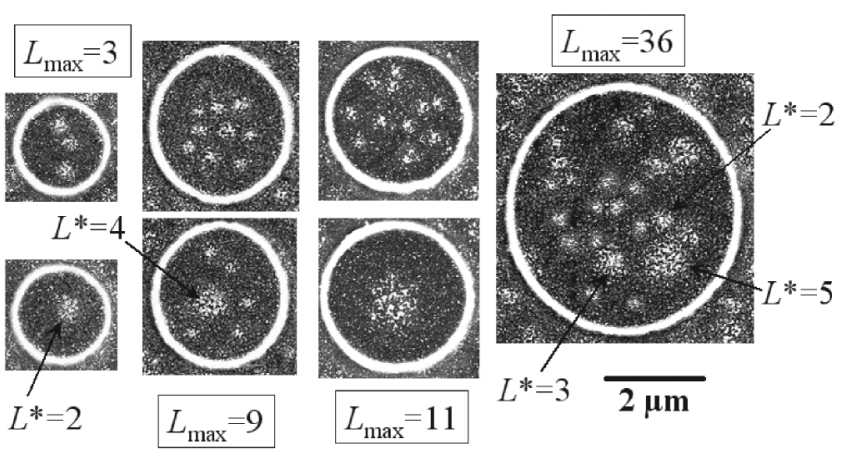

FIG. 2. Vortex configurations in disks with strong disorder for different values of $L_{\max }$ (see text). $L^{*}$ is the vorticity of GVs or clusters estimated from their diameters.

merged into GVs or clusters with vorticity $L^{*} \geq 2$ (as explained below, a single-core GV and a multicore cluster with the same $L$ have the same magnetic size and therefore cannot be distinguished by Bitter decoration). We emphasize that, although the presence of strong disorder appears to be the necessary condition for the formation of GVs or clusters, they are only formed in small disks and were never observed in the bulk of the same crystals (away from the disk arrays). To estimate $L^{*}$ associated with different clusters or GVs we analyzed intensity profiles of many different vortex images; see Fig. 3. Here we distinguish between the results obtained on disks containing only individual, only merged, or a combination of individual and merged vortices. One can see that (i) the size of individual vortices is well defined and shows only small variations between different disks and/or the bulk, and (ii) merged vortices show only a few typical sizes (rather than a continuous distribution), with the same sizes found in disks with only one merged vortex (where the vorticity can be determined fairly unambiguously from $L$ vs $\Phi / \Phi_{0}$ curves) and in disks containing combinations of individual and merged vortices. This allowed us to identify the number of vortices merged in different clusters or GVs, $L^{*}$, as shown in Fig. 3. In the example of the disk in Fig. 3, this yields five individual vortices and one cluster $(\mathrm{GV})$ with $L^{*}=4$; i.e., total vorticity $L=9$, in agreement with $L$ expected for the corresponding $\Phi / \Phi_{0}$ value and with the number of vortices observed in the same experiment in disks with only individual vortices. Similar agreement was found for our largest $(5 \mu \mathrm{m})$ disks, such as that in Fig. 2. According to the measured vortex sizes, here we observe 16 individual vortices with $L^{*}=1$, three merged vortices with $L^{*}=2$, two merged vortices with $L^{*}=3$, and one vortex with $L^{*}=5$. The total vorticity is then $L=33$, again in agreement with $\Phi / \Phi_{0}$.

To model the role of pinning in a confined geometry theoretically, we place a superconducting disk of thickness $d$ and radius $R$ in a perpendicular external field $\boldsymbol{H}$. The forces of vortex interactions with each other $\mathbf{f}_{i}^{v v}$ and with the shielding currents and the edge $\mathbf{f}_{i}^{s}$ can then be modeled as $\quad[9,10] \quad \mathbf{f}_{i}^{v v}=f_{0} \Sigma_{k=1}^{L}\left\{\left(\mathbf{r}_{i}-\mathbf{r}_{k}\right) /\left|\mathbf{r}_{i}-\mathbf{r}_{k}\right|-r_{k}^{2}\left(r_{k}^{2} \mathbf{r}_{i}-\right.\right.$

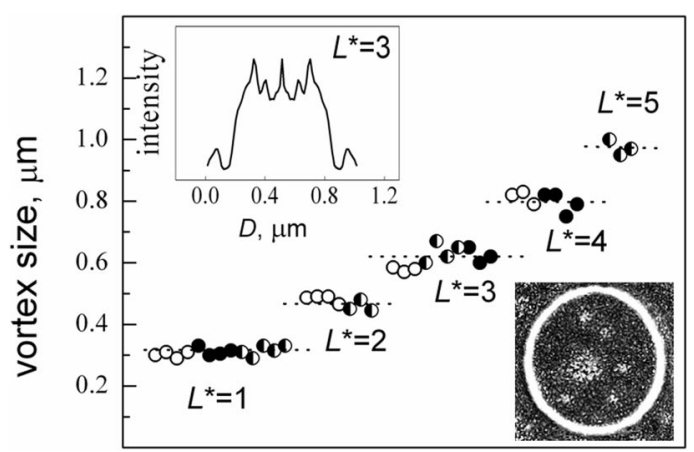

FIG. 3. Diameters of individual and merged vortices found from image intensity profiles on disks containing only one vortex, either individual or merged $(\bigcirc)$, one merged and several individual vortices, as in the bottom-right inset $(\mathbf{)})$, a mixture of individual and merged vortices of different sizes, as in the image of Fig. 2 for $L_{\max }=36$ (half solid, half open circle). Top-left inset shows a typical radially averaged intensity profile for a vortex image. $L^{*}$ are estimated values of vorticity for clusters of different diameters

$\left.\left.\mathbf{r}_{k}\right) /\left|r_{k}^{2} \mathbf{r}_{i}-\mathbf{r}_{k}\right|\right\}$ and $\mathbf{f}_{i}^{s}=f_{0}\left\{1 /\left(1-r_{i}^{2}\right)-h\right\} \mathbf{r}_{i}$, where $h=\pi R^{2} \mu_{0} H / \Phi_{0}=\left(H / 2 H_{c 2}\right)(R / \xi)^{2}, \mathbf{r}_{i}=\boldsymbol{\rho}_{i} / R$ is the position of the $i$ th vortex, $L$ the vorticity, and $f_{0}=$ $4 \pi \mu_{0} \xi^{2} H_{c}^{2} / R$ the unit of force. Our numerical approach is based on the Langevin dynamics algorithm, where the time integration of the equations of motion is performed in the presence of a random thermal force. Then the overdamped equations of motion become $\eta \mathbf{v}_{i}=\mathbf{f}_{i}=\mathbf{f}_{i}^{v v}+$ $\mathbf{f}_{i}^{v p}+\mathbf{f}_{i}^{T}+\mathbf{f}_{i}^{s}$. Here $\mathbf{f}_{i}^{v p}$ is the force due to vortex-pin interactions (see, e.g., [11,12]) which is modeled by shortrange parabolic potential wells located at positions $\mathbf{r}_{k}^{(p)} ; \eta$ is the viscous Bardeen-Stephen friction, which we take $\eta=1$. The pinning force is $\mathbf{f}_{i}^{v p}=\Sigma_{k=1}^{N_{p}}\left(f_{p} / r_{p}\right) \mid \mathbf{r}_{i}-$ $\mathbf{r}_{k}^{(p)} \mid \Theta\left(r_{p}-\left|\mathbf{r}_{i}-\mathbf{r}_{k}^{(p)}\right| \mathbf{r}_{k}\right) \mathbf{r}_{i k}^{(p)}$, where $N_{p}$ is the number of pinning sites, $f_{p}$ the maximum pinning force for each potential well, $r_{p}$ the pinning range (for strong disorder some pinning centers can overlap, effectively forming a single pinning site with an enhanced energy profile), $\Theta$ the Heaviside step function, and $\mathbf{r}_{i k}^{(p)}=\left(\mathbf{r}_{i}-\mathbf{r}_{k}^{(p)}\right) /\left|\mathbf{r}_{i}-\mathbf{r}_{k}^{(p)}\right|$. The $\mathbf{f}_{i}^{T}$ is the thermal stochastic force satisfying $\left\langle\mathbf{f}_{i}^{T}(t)\right\rangle=0$ and $\left\langle\mathbf{f}_{i}^{T}(t) \mathbf{f}_{j}^{T}\left(t^{\prime}\right)\right\rangle=2 \eta \delta_{i, j} \delta\left(t-t^{\prime}\right) k_{B} T$. The ground state of the system is obtained by simulating field-cooled experiments. We now consider a disk containing, e.g., 8 vortices in a field $h=15$ (for $R=1.5 \mu \mathrm{m}$, this corresponds to $H \approx 45 \mathrm{Oe}$ ). For a perfect disk (no pinning), vortices form a symmetric two-shell configuration $(1,7)$ (cf. [3]) as shown in Fig. 4(a). A weak-pinning site placed near the disk's center distorts the symmetric configuration, moving away the central vortex; the rest of the vortices adjust themselves accordingly [Fig. 4(b)]. However, the situation changes dramatically if $f_{p}$ is increased: (i) a stronger pin traps 3 out of 8 vortices resulting in vortex merger into a cluster with $L=3$ coexisting with individual vortices [Fig. 4(c)], similar to the experimental pattern shown in Fig. 3. A stronger still pin traps 5 out of 8 vortices 


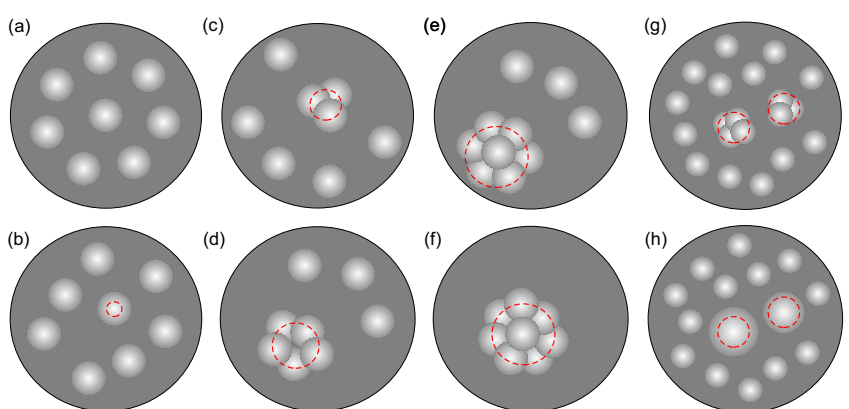

FIG. 4 (color online). Vortex patterns for $L=8$ (a)-(d), $L=10$ (e),(f), and $L=20$ (g),(h) in disks with different pinning potentials. (a) No pinning. (b) Weak pinning, $f_{p} / f_{0}=2, r_{p}=$ 0.08 ; dashed line shows the pinning range. (c), (d) Strong pinning, $f_{p} / f_{0}=4, r_{p}=0.16$, and $f_{p} / f_{0}=6, r_{p}=0.24$, respectively; combination of merged and individual vortices. (e),(f) $f_{p} / f_{0}=8, r_{p}=0.32$ : (e) Pinning site close to the boundary, combination of merged vortices with $L=7$ and 3 individual vortices; (f) enhancement of pinning strength for a pin near the center: $L=8$ vortices are merged, while other vortices are expelled from the disk by strong repulsion from the cluster. (g),(h) Formation of multiquanta vortices for $f_{p} / f_{0}=10, r_{p}=$ 0.16 , and different $r_{\min }:(\mathrm{g}) r_{\min }=0.048$, vortices form clusters with $L=5$ (near center) and $L=4$ (out of center); (h) $r_{\min }=0.064$, GVs with $L=5$ (near center) and $L=4$ (out of center).

[Fig. 4(d)]. Furthermore, we find that the ability of a pin to trap vortices depends on its position inside a mesoscopic disk: a pin close to the disk's center traps more vortices than the same pin near the boundary [see Figs. 4(e) and 4(f)], i.e., its pinning strength is enhanced when it is near the center. (ii) The total vorticity $L$ in a disk with strong pins is lower than in the weak-pinning situation because vortices are repelled by a cluster stronger than by a single vortex, pushing them towards the disk boundary so that some vortices leave the sample. This explains the observed enhanced diamagnetic response of strong-disorder disks (see Fig. 1). To clarify whether the above vortex merger corresponds to the formation of true GVs with a single core or multiquanta vortex clusters, we calculated the distributions of the order parameter $|\Psi|^{2}$ and the phase $\varphi$ using the Ginzburg-Landau (GL) equations. It is known $[1,2]$ that in perfect disks without pinning GVs appear as a ground state only if the disk's radius is small enough. For the disk sizes studied here, the ground state in the absence of pinning corresponds to vortex shells (MVS) as observed in Ref. [3]. However, if vortices are trapped by a strong potential they can merge, forming a GV, because repulsive vortex-vortex interaction (logarithmic at small distances [13]) vanishes at very small distances $r_{\min }$ when vortex cores strongly overlap [14]. To demonstrate formation of GVs in disks with strong pinning, we introduced a pinning potential $U_{\text {pin }}(\rho)$ in the dimensionless GL equations (see, e.g., [12]): $(-i \boldsymbol{\nabla}-\mathbf{A})^{2} \Psi=\Psi\left[1-U_{\text {pin }}(\rho)-|\Psi|^{2}\right]$, where $U_{\text {pin }}=$ $U_{0} \exp (-\rho / w)$, and $\rho=\sqrt{\left\{\left(x-x_{\mathrm{displ}}\right)^{2}-\left(y-y_{\mathrm{displ}}\right)^{2}\right.}$.
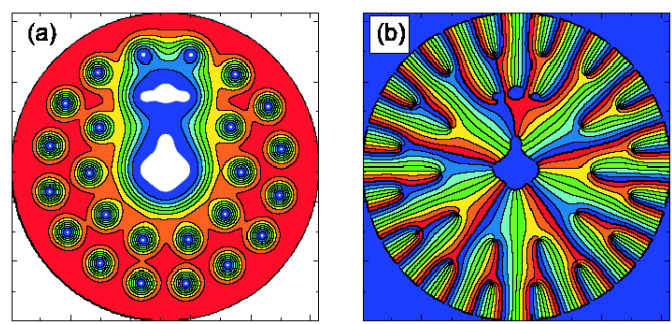

FIG. 5 (color online). (a) Cooper pair density $|\Psi|^{2}$ in a disk with $R=20 \xi, H=0.2 H_{c 2}$, for two pinning sites with $U_{0}=1$, and $w=5 \xi$, at the center and out of center. (b) Phase pattern corresponding to (a) showing a GV with $L=5$ (center) and a cluster with $L=3$ (out of center).

The results of calculations of $|\Psi|^{2}$ and $\varphi$ are shown in Figs. 5(a) and 5(b) for a disk with $R=20 \xi$ and pins with $U_{0}=1$ and $w=5 \xi$ at the center and out of center, respectively. The Cooper pair density $|\Psi|^{2}$ vanishes at pin positions, forming large spots (larger than individual vortices): The central spot is a GV with vorticity $L=5$, while the one at the distance of a half of the disk's radius is a cluster consisting of $L=3$ individual vortices. Thus, depending on the pinning strength, range, and $r_{\min }$ (e.g., $r_{\min }>50 \mathrm{~nm}$ for disks with $R=1 \mu \mathrm{m}, f_{p} / f_{0}=10, r_{p}=$ 0.16, as shown in Figs. 4(g) and 4(h), where the lengths are in units of $R$ ), vortices can form either clusters [Fig. 4(g)] or GVs with $L=5$ (center) and $L=4$ (out of center), Fig. 4(h). We also analyzed magnetic sizes of single-core GVs and multicore clusters of the same $L$ and found them to be practically identical (which makes it impossible to distinguish them experimentally using a magnetic-field sensitive technique). We note, however, that the above pinning forces are of the same order of magnitude as $f_{p}$ estimated for $\geq \xi$ normal inclusions in $\mathrm{Nb}$ [15], with pins larger than $100 \mathrm{~nm}$ having $f_{p}$ and $r_{p}$ sufficient to form a true GV.

*Corresponding author.

[1] V. A. Schweigert et al., Phys. Rev. Lett. 81, 2783 (1998).

[2] A. Kanda et al., Phys. Rev. Lett. 93, 257002 (2004).

[3] I. V. Grigorieva et al., Phys. Rev. Lett. 96, 077005 (2006).

[4] C. Veauvy et al., Phys. Rev. B 70, 214513 (2004).

[5] W. DeSorbo, Phys. Rev. 132, 107 (1963).

[6] N. Norman, J. Less-Common Met. 4, 52 (1962); H. Safa et al., J. Alloys Compd. 232, 281 (1996).

[7] I. V. Grigorieva, Supercond. Sci. Technol. 7, 161 (1994).

[8] A. Bezryadin et al., Phys. Rev. B 53, 8553 (1996).

[9] A. I. Buzdin and J. P. Brison, Phys. Lett. 196A, 267 (1994).

[10] B. J. Baelus et al., Phys. Rev. B 69, 064506 (2004).

[11] C. Reichhardt et al., Phys. Rev. Lett. 78, 2648 (1997).

[12] V. R. Misko et al., Phys. Rev. Lett. 95, 177007 (2005).

[13] M. Tinkham, Introduction to Superconductivity (McGrawHill, New York, 1996).

[14] E.-H. Brandt, J. Low Temp. Phys. 53, 41 (1983).

[15] A. M. Campbell and J.E. Evetts, Adv. Phys. 21, 199 (1972). 